\title{
Mathematical Modeling and Quantitative Analysis of the Demographic and Ecological Aspects of Russian Supermortality
}

\author{
A.K. Cherkashin ${ }^{1 *}$ and Ya.A. Leshchenko ${ }^{2}$ \\ ${ }^{1}$ V.B. Sochava Institute of Geography SB RAS, Irkutsk, Russia \\ ${ }^{2}$ Angarsk Branch of the East-Siberian Center for Human Ecology SB RAMS \\ Research Institute of Labor Medicine and Human Ecology, Angarsk, Russia
}

\begin{abstract}
We have carried out a polysystem analysis of the population dynamics by using a variety of hypotheses and their respective models based on different system interpretations of the phenomenon under investigation. Each of the models supplements standard dynamic equations for explaining the effects observed. A qualitative model-based analysis is made of the age-specific male mortality for a Siberian industrial city. The study revealed the tendencies for background mortality to increase with age and over time, which characterizes in an integral manner the influence of socio-ecological factors on the decline in population viability. It is shown that these tendencies are similar for different years and for different population age groups.
\end{abstract}

Key words: demographic crisis, mortality, socio-ecological factors, mathematical modeling, data analysis

AMS subject classification: 91D20

\section{Introduction}

When investigating the medico-demographic and demoecological processes, it is important to take into account the features of the socio-ecological situation and temporal variability of its characteristics, revealing essential indicators of the situation and tendencies toward its change. The high variability of demographic and social phenomena and of their interconnections restricts the possibilities of implementing standard statistical procedures for data processing that shaped themselves

${ }^{*}$ Corresponding author. E-mail: cherk@mail.icc.ru 
in a homogeneous natural and socio-economic environment. To accomplish this requires special demoecological analysis built upon consecutively extracting from observational evidence a more sophisticated, not infrequently hidden, knowledge of the regularities inherent in the process under study, for assessing situations in different places and at different instants of time by the use of various mathematical models.

Mortality is one of the important demographic indicators responding to the state of external and internal life environment of the human population. A large number of models, based on different hypotheses and describing the influence of the internal [1,2] and external [3] reasons behind the changes in mortality, were suggested. It is of interest to trace, on the basis of the particular data, the tendencies toward change in response (the mortality indices) on the integral impact of the various factors and conditions.

In the post-Soviet period (since 1992), population mortality in Russia has been at a very high level, far exceeding the respective indices of all developed and moderately developed (industrial) countries. This fact has often been characterized by Russian and foreign researchers as Russian supermortality, or Premature Death [4]. The literature on this phenomenon is quite extensive, although it differs greatly in interpretation by different authors, which in itself is testimony to the complicated multifactor nature of the phenomenon, and to its being poorly explored.

\section{Model and methods}

\subsection{Object of investigation}

The study of medico-demographic and demoecological processes is based on information regarding the age pattern of the population, indices of sickness rate, birthrate, mortality, migration, and other indices characterizing, in the aggregate, population reproduction in the quantitative and qualitative context under geographical environment change. An investigation along these lines has been made using the city of Angarsk (Irkutsk region, Russia) as an example, based on statistical data spanning the time interval from 1980 to 2006. Angarsk was selected as the object for investigation, because a monitoring of the medico-demographic processes (specifically of the mortality caused by various factors) using the computerized information system had been carried out in this city by the Angarsk Branch of the East-Siberian Scientific Center for Human Ecology SB RANS over the course of nearly 30 years.

Angarsk is among the cities of new industrial development which were constructed after World War II for the purpose of promoting development of the defense-industrial capacity in the USSR's eastern regions. The city was founded in 1951 in East Siberia, on the left bank of the Angara river $46 \mathrm{~km}$ to the north-west of the regional center, the city of Irkutsk; it is an urbanistic entity at the industrial and power generating complex. The population of Angarsk had increased to 267-268 thousand by 1992-1995, and the population size started to decline slowly thereafter. In 2008, the number of inhabitants of the city was 242.5 thousand.

Starting in 1992, the liberal socio-economic reforms in the Russian Federation were accompanied by a system crisis implying a dramatic decline in the living standard of the population, a social maladaptation and psychosocial stress state of humans, difficulties in ensuring adequate 
labor-based conditions of life, the high degree of property stratification, loss of social protectedness and favorable outlook for life, a critical change for the worse in the operation of the system of medical assistance to the population, criminalization of all aspects of the life of society and loss of personal security, and, finally, a spiritual crisis [5-8]. These factors have all affected the population mortality indices which started to increase precipitously. In Angarsk, the aforementioned processes were occurring in full accord with the nationwide tendencies and regularities, and this permitted the city to be treated as a model object in carrying out demoecological investigations.

Mortality as a crucial medico-demographic characteristic furnishes a means of determining the worsening of the populations sanitary state, and a change in public health under the influence of the living conditions and lifestyle. The lethal level of the population is the result of a functional synthesis of endogenous ontogenetic factors with exogenous factors of natural and social environment. Our investigation into mortality of the population of Angarsk covers a 27-year-long period (1980-2006) and is based on medico-ecological monitoring data.

According to results from different investigations, the factors of social trouble to a greater extent influence the mortality indices of the male population when compared with the female population [7-8]. The lethal level for males increases with the worsening of the conditions and increases with their improvement to a greater extent than does the lethal level for females. Medicodemographic monitoring data indicate that the index of total mortality for males in Angarsk exceeds the female mortality nearly by a factor of 1.5. It began to increase in 1992 to reach the value 15.5 in 1994 (Fig. 1). Following a slight decline in 1996-1997, it increased again to reach by the year 2006 a still higher value, 19.7. Since the male population is more sensitive to the influence of the socio-ecological factors than is there female population, age-specific mortality indices for the city's male population were used in the quantitative analysis.

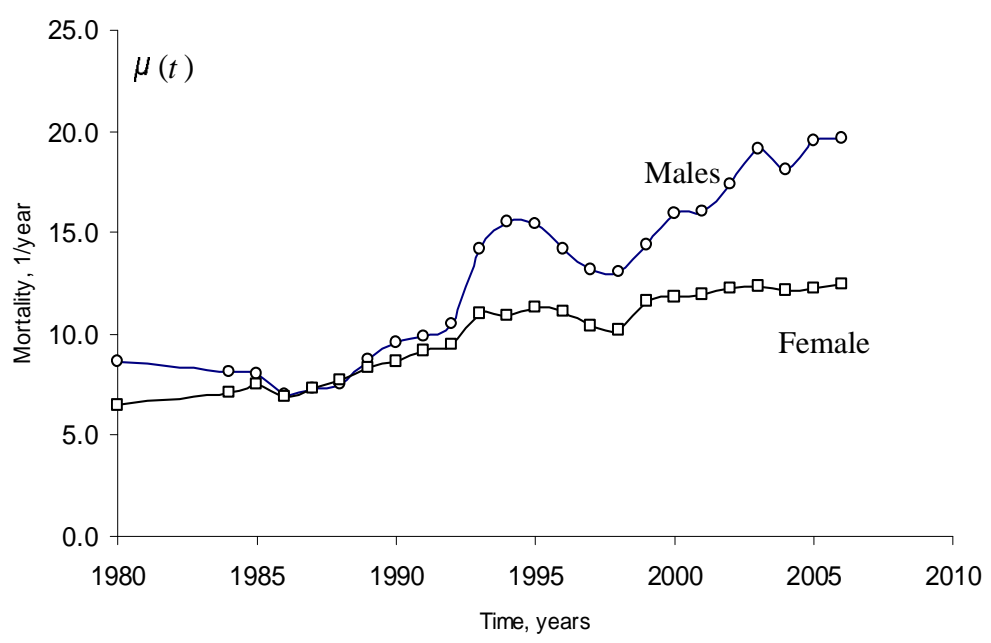

Figure 1: Variation in total mortality indices for males and females in the city of Angarsk during 1980-2006 (per 1000 residents) 


\subsection{Methodological approaches and principles of investigation}

The consequences caused by the influence of the totality of socio-ecological factors were assessed from the changes in the mortality index $\mu(t, \tau)$, the number of the deceased for a year for various reasons in age group $\tau$ at time $t$ (year) per 100 thousand in the group. In the case of no migration, the dynamics of the population size $N(t, \tau)$ is described by the well-known differential equation $\frac{\partial N}{\partial t}+\frac{\partial N}{\partial \tau}=-\mu(t, \tau) N(t, \tau)$, representing a variation of $N(t, \tau)$ as a result of the transition of humans from one age group to the other with time $t$, and the dying out of a part of the population for a variety of reasons in different age groups $\mu(t, \tau) N(t, \tau)$. This equation is rearranged to give

$$
\text { a) } \left.-\mu(t, \tau)=\frac{1}{N(t, \tau)} \frac{\partial N}{\partial t}+\frac{1}{N(t, \tau)} \frac{\partial N}{\partial \tau} ; b\right)-\mu(t, \tau)=\frac{\partial \ln N}{\partial t}+\frac{\partial \ln N}{\partial \tau}
$$

According to (2.1a), the value of $\mu(t, \tau)$ equals the sum of relative variation rates of $N(t, \tau)$ with time $t$, and with age $\tau$. Equation (2.1b) is obtained from (2.1a), because $\frac{\partial \ln N}{\partial t}=\frac{1}{N} \frac{\partial N}{\partial t}$. The solution to the differential equations is determined for the initial conditions $N(t, \tau)=N(0, \tau)$, and for the boundary conditions $N(t, \tau)=N(t, 0)$, representing the number of new-born children in year $t$.

Investigations for assessing the influence of environmental factors on population mortality and lifetime make wide use of the Gompertz-Makeham equation [9]:

$$
\mu(t, \tau)=A(t)+B(t) \exp [\alpha(t) \tau]
$$

where $A(t)$ is the background mortality coefficient characterizing the socio-ecological component of mortality; $B(t)$ is the initial value of a dependence of the mortality rate on age $\mu(t, 0)=$ $A(t)+B(t)$, and $\alpha(t)$ is the relative growth rate of mortality with age (the ageing rate of a population). Formula (2.2) consists of two components: an integral estimation of the influence of the socio-ecological environment $A(t)$, and the influence $B(t) \exp (\alpha(t) \tau)$ ) of the natural-biological factor, i.e. age $\tau$. The biological component shows how the population mortality rate in the age range 30-80 years increases exponentially with a decrease in viability potential (agieng) [9]. The research reported in [9-10] demonstrated that in the 20th century the increase in life expectancy of the population in the developed countries was determined solely by the decrease of the socioecological component of mortality $A(t)$.

Demographic dynamics is a complex process necessitating appropriate description tools. A characteristic feature of the demoecological approach is a multiaspect reflection of processes and phenomena, which leads to their polysystem interpretation [11-13] where the object is regarded as a polysystem, the result of a real interaction of systems of a different kind. For each type of systems, its own theory and respective, mutually complementing, types of models are developed, so that a dynamic model of the form (2.1) is a nonunique interpretation of the phenomenon under study. Such a formulation of research is closely allied to the concept of multiple working hypotheses [14], and, in the present state of the art, it has been realized through the creation of multimodels [15-17].

Several currently important areas of research into the processes of functioning and development of demoecological systems (DES) are recognized, which are related to the present-day demographic situation in Russia: 1) identification of the main types of models and theories explaining 
the DES; 2) generation of the space of models, and identification of the position of the DES in this space; 3) creation of knowledge synthesis models for different theoretical interpretations of the DES; 4) system analysis of models with the purpose of elucidating the regularities inherent in the transformation of one state of the DES to another, and 5) searching for ways of achieving an optimal development of the DES with limited resources. The focus in this paper is on area 1 and 4.

\section{Results}

\subsection{Theoretical analysis}

The emergence of the phenomenon of population supermortality cannot be attributed solely to the decline in the level of material well-being, as this would immediately affect the mortality dynamics of such vulnerable (ecosensitive) groups of population as children and elderly people. A decisive contribution to the decline in lifetime during the 1990-2000s was made by the increase in mortality of the most able-bodied part of the population, the males at the age between 30 and 60 years (Table 1), and to a lesser extent, the females at the age from 30 to 75 years [18-21]. Noteworthy in this case is the rapid growth rate of mortality, and the low conditionality of this increase by the factors associated with undernourishment, the worsening of the housing conditions, with infectious diseases, etc. The main increase in mortality, however, is caused by so-called unnatural (external) factors and circulatory diseases. This phenomenon is customarily explained by: 1) the increased level of phsychosocial stress in society due to the deterioration of the economic situation of the population [19-26]; 2) disorder of the dynamic stereotype of higher nervous activity [5, 7, 19, 25-26], which, we believe, is at the basis of social maladaptation, and 3) the increase in spiritual trouble, an important manifestation of which is social depression which is determined indirectly from the level of suicides [6-8]. Variation in age indices for males in the city of Angarsk as caused by all factors (per 100000 males of a given age group)

Models of psychosocial stress have two system-theoretical components: a) the decrease of susceptibility (sensibility) thresholds to environmental impacts, and b) disorder of the adaptation mechanisms of living organisms to disturbances in the environment.

The level of stressor response $E_{c}$, like the other emotions, is determined from the necessitiesand-information model suggested by P.V. Simonov [27-28] on the basis of the concepts of humans or a community regarding the amount of necessities $R$ (the desired) and the possibility $R_{0}$ (the real) of satisfying them:

$$
E_{c}=f\left(k, R_{0}-R\right) \approx k\left(R_{0}-R\right),
$$

where $k$ is the index (coefficient, factor) of force and quality of the actual necessities, and $f(\cdot)$ is the formula of emotions having an obvious property $\frac{\partial f}{\partial\left(R_{0}-R\right)} \geq 0$, for instance, when $R_{0} \geq R$ (the real is larger than the desired) there arise positive emotions $(k>0)$. The inversion of the direction of emotional response $\left(\frac{\partial f}{\partial\left(R_{0}-R\right)} \leq 0, k>0\right)$ disturbs the natural regulation mechanisms where the predominance of the possibilities over the desired living standard leads to a negative response, a 
Table 1: Variation in age indices for males in the city of Angarsk as caused by all factors (per 100 000 males of a given age group)

\begin{tabular}{|c|c|c|c|c|c|c|c|c|c|c|}
\hline & \multicolumn{10}{|c|}{ Calendar years } \\
\hline Age, years & 1986 & 1987 & 1988 & 1990 & 1991 & 1992 & 1993 & 1994 & 1995 & 1996 \\
\hline 0 & 2057 & 1689 & 2185 & 2439 & 2013 & 1557 & 2038 & 1665 & 1884 & 1945 \\
$1-4$ & 63 & 48 & 91 & 131 & 74 & 66 & 201 & 128 & 161 & 133 \\
$5-9$ & 61 & 40 & 39 & 48 & 67 & 38 & 57 & 58 & 89 & 134 \\
$10-14$ & 18 & 87 & 41 & 52 & 72 & 60 & 59 & 39 & 86 & 86 \\
$15-19$ & 100 & 75 & 108 & 158 & 191 & 279 & 301 & 246 & 316 & 310 \\
$20-24$ & 116 & 104 & 109 & 211 & 148 & 170 & 284 & 382 & 380 & 614 \\
$25-29$ & 196 & 168 & 204 & 243 & 207 & 392 & 494 & 447 & 449 & 402 \\
$30-34$ & 225 & 258 & 201 & 354 & 394 & 453 & 612 & 729 & 721 & 597 \\
$35-39$ & 352 & 336 & 337 & 405 & 608 & 608 & 852 & 1103 & 918 & 739 \\
$40-44$ & 403 & 359 & 520 & 585 & 834 & 954 & 1282 & 1449 & 1491 & 1153 \\
$45-49$ & 851 & 1028 & 878 & 1073 & 740 & 1498 & 1732 & 2244 & 1880 & 1533 \\
$50-54$ & 1116 & 1002 & 1136 & 1482 & 1558 & 1499 & 2420 & 2679 & 2766 & 2369 \\
$55-59$ & 1828 & 2379 & 1984 & 2293 & 2087 & 2306 & 3143 & 3535 & 3154 & 2761 \\
$60-64$ & 2680 & 3173 & 2689 & 3793 & 3591 & 3650 & 4681 & 5107 & 4285 & 3961 \\
$65-69$ & 3844 & 4430 & 4843 & 4238 & 6201 & 4985 & 5893 & 6267 & 6425 & 6248 \\
$70-74$ & 7407 & 5474 & 6071 & 7834 & 5941 & 7577 & 9539 & 9354 & 7794 & 8374 \\
$75-79$ & 11893 & 7433 & 9052 & 13883 & 11111 & 9602 & 13899 & 12778 & 12881 & 11085 \\
$80-84$ & 12722 & 15339 & 16022 & 20103 & 18272 & 16312 & 19865 & 17241 & 20588 & 17540 \\
$>84$ & 20472 & 24818 & 18792 & 17919 & 20283 & 22422 & 29878 & 31532 & 23757 & 24752 \\
Total & 700 & 719 & 746 & 961 & 985 & 1054 & 1424 & 1569 & 1536 & 1424 \\
\hline
\end{tabular}

\begin{tabular}{|c|c|c|c|c|c|c|c|c|c|c|}
\hline Age, years & 1997 & 1998 & 1999 & 2000 & 2001 & 2002 & 2003 & 2004 & 2005 & 2006 \\
\hline 0 & 1711 & 1710 & 1122 & 962 & 1130 & 835 & 1533 & 693 & 555 & 1391 \\
$1-4$ & 120 & 20 & 63 & 44 & 23 & 23 & 91 & 86 & 41 & 20 \\
$5-9$ & 33 & 72 & 40 & 0 & 46 & 32 & 17 & 69 & 18 & 72 \\
$10-14$ & 123 & 29 & 67 & 20 & 92 & 77 & 24 & 26 & 28 & 75 \\
$15-19$ & 292 & 207 & 194 & 289 & 296 & 278 & 165 & 198 & 224 & 175 \\
614 & 457 & 548 & 778 & 887 & 656 & 446 & 497 & 694 & 687 & 452 \\
$25-29$ & 410 & 391 & 484 & 783 & 770 & 688 & 638 & 945 & 1076 & 588 \\
$30-34$ & 676 & 577 & 408 & 591 & 553 & 631 & 769 & 1048 & 1098 & 856 \\
$35-39$ & 642 & 735 & 751 & 719 & 843 & 1106 & 1173 & 999 & 1344 & 830 \\
$40-44$ & 926 & 1032 & 1002 & 1095 & 1285 & 1560 & 1759 & 1444 & 1858 & 1277 \\
$45-49$ & 1357 & 1368 & 1611 & 1604 & 1654 & 1714 & 2102 & 1934 & 2242 & 1755 \\
$50-54$ & 1911 & 1820 & 1969 & 2398 & 2402 & 2737 & 3534 & 2825 & 2837 & 2520 \\
$55-59$ & 2847 & 2566 & 2799 & 3550 & 3256 & 3330 & 3796 & 3339 & 3557 & 3391 \\
$60-64$ & 3073 & 3669 & 3811 & 4501 & 4527 & 5006 & 5684 & 5322 & 5088 & 4392 \\
$65-69$ & 5474 & 5499 & 5651 & 5658 & 5485 & 5894 & 6021 & 5725 & 6076 & 5791 \\
$70-74$ & 8420 & 7184 & 8204 & 8471 & 7933 & 8679 & 8555 & 7383 & 7648 & 7451 \\
$75-79$ & 11175 & 11526 & 12387 & 11396 & 11060 & 9815 & 10131 & 9325 & 10371 & 10822 \\
$80-84$ & 17209 & 14489 & 17689 & 14351 & 13739 & 13652 & 11669 & 12245 & 11451 & 9387 \\
$>84$ & 27411 & 20524 & 21277 & 35096 & 26633 & 24180 & 23166 & 16139 & 5285 & 14333 \\
Total & 700 & 1305 & 1441 & 1590 & 1597 & 1742 & 1914 & 1809 & 1950 & 1700 \\
\hline
\end{tabular}


stress. The emotions themselves determine the direction of actions

$$
E_{c}=-\frac{d\left(R_{0}-R\right)}{d t}
$$

minimizing (by the Le Chatelier-Braun principle) the differences of $R$ and $R_{0}$ with saturation:

$$
\frac{d\left(R_{0}-R\right)}{d t}=-k\left(R_{0}-R\right) .
$$

Equations (3.3) represent the simplest adaptive model of behavior focusing on satisfaction of necessities, and on regulation of the state of an organism or a population. When $k>0$ the behavior is stable, tending to an equilibrium $R=R_{0}$, and when $k<0$ it is unstable, with a constant increase or decrease in characteristics of the state of the system, which is implied in, for example, the law of "rising necessities" or a regular increase in mortality with age.

Now, if the regulation model is extended to population variability and if it is assumed that $R=\ln N(t, \tau)$ ) and $R_{0}=\ln N_{0}(t, \tau)$ ), then from (3.3) we obtain the dynamic equations of the population size and of change of the mortality coefficient:

a) $\frac{d}{d t}\left(\ln N_{0}-\ln N\right)=-k\left(\ln N_{0}-\ln N\right)$, then $\left.b\right) \ln \left(\ln N-\ln N_{0}\right)=-k t+\ln C$,

Here $N(t, \tau)$ and $N_{0}(t, \tau)$ are the real and potential population size, respectively, which depends on the entire complex of environmental factors and generally varies with time and age. The value of $C(\tau)=\ln \left[N_{H}(\tau) / N_{0}(0, \tau)\right]$ is determined by the initial distribution $N(0, \tau)=N_{H}(\tau)$ of the population in age. Hence, when $A(t)=\mu_{0}(t)$ (background mortality) equation (3.4d) is an analogue of the Gompertz - Makeham equation (2.2) and describes the time variation of the population size of separate age groups (when $k>0$ ) along the stabilization direction at the level $N(\infty, \tau)=N_{0}(\tau)$.

An identical equation holds true for the age dynamics

$$
\ln N=C \exp (-k \tau)+\ln N_{0},
$$

where $C(t)=\ln \left[N_{0 H}(t) / N_{0}(t, 0)\right]$, and $N(t, 0)=N_{0 H}(t)$ is the birthrate at time $t$. Equation (3.5) when $k<0$ describes the variation in the size of a population group of the same birth year with age.

The regulation equation (3.3) for the case of both temporal and age variability has the form:

$$
\text { a) } \frac{\partial\left(R_{0}-R\right)}{\partial t}+\frac{\partial\left(R_{0}-R\right)}{\partial \tau}=-k\left(R_{0}-R\right), \quad \text { b) } \frac{\partial \ln \left(N / N_{0}\right)}{\partial t}+\frac{\partial \ln \left(N / N_{0}\right)}{\partial \tau}=-k \ln \left(N / N_{0}\right)
$$

One of the possible solutions to equation (3.6b) is

$$
\ln N(t, \tau)=\ln N_{0}(t, \tau)+C \exp [c t-(c+k) \tau],
$$

where $C$ and $c$ are, in this case, constants of integration that govern the situation. When $c=0$, equation (3.7) transforms to (3.5), and when $c=-k$ it changes to (3.4c). When $c<0$ there is 
taking place a decrease in mortality with time, and when $c>0$ we have an increase in mortality characteristic for the Russian conditions.

The dynamic equation in the form (2.1a) is comparable with the regulation equation (3.6b), if in (2.1) we pass from $\mu(t, \tau)$ and $N(t, \tau)$ to relative indices of functioning $\mu_{1}(t, \tau)=\mu(t, \tau)-\mu_{0}(t, \tau)$ and structure $N(t, \tau) / N_{0}(t, \tau)$. Then, according to (3.6b) and (3.7), we have

$$
\mu_{1}(t, \tau)=\mu(t, \tau)-\mu_{0}(t, \tau)=k \ln \left[N(t, \tau) / N_{0}(t, \tau)\right]=-k C \exp [c t-(c+k) \tau],
$$

i.e. the laws of structure (3.7) and functioning (3.8) are identical in this case. Then equation (3.6b) holds both for $\ln \left(N / N_{0}\right)$ and for $\mu_{1}(t, \tau): \frac{\partial \mu_{1}}{\partial t}+\frac{\partial \mu_{1}}{\partial \tau}=-k \mu_{1}$. Only in this case are the dynamic model (2.1) and the regulation model (2.1) comparable to the population size $N(t, \tau)$ varying with time and age. Furthermore, we would be more correct in considering the dynamic model (2.1) on a per-unit basis $N / N_{0}$, i.e. in counting the population size $N(t, \tau)$ in comparison with the potential $N_{0}(t, \tau)$ of environment, the value to which $N(t, \tau)$ tends.

When $c=0$ and $\alpha=-k>0$, equation (3.8) corresponds to the Gompertz - Makeham equation (2.2), and when $c=-k$ we have as strictly temporal dependence of mortality. In the general case, $c$ has a variety of values, and for the territory under investigation we must have $c>0$ (mortality increases with time, see Fig. 1), and $c+k<0,0<c<-k, k<0$ (mortality increases with age). Thus, according to equations (3.6) - (3.8), the situation of a substantial increase in mortality which we consider here (see Fig. 1) is theoretically possible. In the norm, it must be assumed that the mortality rate in an age group is not to increase $(c \leq 0)$, whereas the lifetime is to increase.

When using the adaptive regulation model for the population size (3.3) and (3.6), the hypothesis of the connection of the population response with impact factors was invoked:

$$
R=\ln N(t, \tau)-\ln N_{0}(t)+R_{0}=\ln \left[N(t, \tau) / N_{0}(t)\right]+R_{0},
$$

where the influence factor is represented by the current population size with due regard for its age pattern and changes over time. The analogue of equation (3.9) is known in science as the empirical psychophysiological Weber - Fachner law [29], according to which the intensity of sensation $y$ is proportional to the logarithm of the intensity of stimulus $x: y=k \ln \left(x / x_{0}\right)+y_{0}$, where $x_{0}$ is the threshold value of the intensity of the irritant (characteristic of the system), and $y_{0}$ is the threshold value of sensation.

Consequently, in deriving the explaining equations, one has to use three types of models: the model of population dynamics, the model of adaptive regulation, and the model of the impact intensity of factors, which makes it possible in (3.9) to take into account, by analogy, the sum of all influencing factors $x_{i}$ :

$$
R-R_{0}=\ln \left(N / N_{0}\right)+\ln \left(x / x_{0}\right), \ln \left(x / x_{0}\right)=\sum_{i} \gamma_{i} \ln \left(x_{i} / x_{0 i}\right),
$$

where $x$ is an integral convolution of the influencing factors $x_{i}$ with the threshold $x_{0 i}$ and the weight $\gamma_{i}$. From (3.10) we obtain

$$
\ln \left(N / N_{0}\right)+\ln \left(x / x_{0}\right)=\ln N-\left[\ln N_{0}-\ln \left(x / x_{0}\right)\right]=\ln N-\ln \widetilde{N}_{0}, \ln \widetilde{N}_{0}=\ln N_{0}-\ln \left(x / x_{0}\right),
$$


i.e. the quantity $\ln N_{0}$, changing to $\ln N_{0}-\ln \left(x / x_{0}\right)$, should be interpreted in all equations as resulting from the influence of a set of variable factors of the system external $x$ and internal $x_{0}$ environment.

The transformation $N_{0} \rightarrow \widetilde{N}_{0}$, when the influence of the factors is changed, can be treated as a replacement of the dynamic stereotype that determines the adaptive behavior of humans. Therefore, the causal impacts should be divided into variable $x(t)$ (factors) and relatively constant $x_{0}$ (threshold conditions), and the value of $\widetilde{N}_{0}$ should be calculated by the formula

$$
\ln \widetilde{N}_{0}=\ln N_{0}-\gamma \ln x+\gamma \ln x_{0},
$$

including both stable $x_{0}$ and changeable $x$ characteristics which, in the aggregate, are seen in the phenomenon of psychosocial trouble of the population. The decrease in potential of the population size $\widetilde{N}_{0}$ is determined, according to (3.12), by the increase in the factor influence or by the decrease in corresponding threshold conditions.

As noted above, two peaks are identifiable in the dynamics of the mortality level, which correspond to 1993-1995 and 2000-2005 (see Fig. 1). Their generation has important differences manifesting themselves in the relationship of the influence of factors and conditions. It seems likely that the first peak of mortality is associated with an abrupt change in lifestyle $x_{0}$, which entailed a massive disturbance of the dynamic stereotype of nervous activity (social maladaptation). The second peak appeared after eight years of the society getting accustomed and adjustment to the new life values. In that period, a lingering psychoemotional stress (an increase in negative impacts $x$ ), probably, became the main model of psychosocial trouble, based on economic factors (poverty of wide sections of society, and low remuneration of labor blocking the motivation of improvement of labor quality and productivity).

\subsection{Empirical analysis}

Medico-demographic monitoring data (see Table 1) offer a means of investigating the changes in mortality intensity $\mu(t, \tau)$ simultaneously from the time and age coordinates using equation (3.8) which we rearrange to the form:

$$
\mu(t, \tau)=\mu_{0}(t, \tau)+c C \exp (c t) \exp [-(c+k) \tau] .
$$

The value of relative mortality $\mu(t, \tau)-\mu_{0}(t, \tau)$ is the product of two functions depending in one case only on $t$, and in the other case only on $\tau$, which permits us to fix one variable and investigate the dependence of $\mu(t, \tau)$ on the other variable, i.e. to consider the data in table 1 separately line by line (time variation) and column by column (for age). For instance, we fix $t$ to obtain an equation of the form (3.8), where $A=\mu_{0}(t, \tau), B=c C \exp (c t), \alpha(t)=-(c+k)$. The following differential equation corresponds to it:

$$
\frac{\partial \mu(t, \tau)}{\partial \tau}=-(c+k)\left[\mu(t, \tau)-\mu_{0}(t, \tau)\right]+\frac{\partial \mu_{0}(t, \tau)}{\partial \tau}=-(c+k) \mu(t, \tau)+(c+k) \mu_{0}(t, \tau)+\frac{\partial \mu_{0}(t, \tau)}{\partial \tau}
$$


relating by a linear dependence the change of $\mu(t, \tau)$ with age to the current value of $\mu(t, \tau)$

$$
\frac{\partial \mu(t, \tau)}{\partial \tau}=C_{1} \mu(t, \tau)+C_{2}, C_{1}-=-(c+k), C_{2}=(c+k) \mu_{0}(t, \tau)+\frac{\partial \mu_{0}(t, \tau)}{\partial \tau} .
$$

From this we have the existence of a linear connection of $C_{1}$ and $C_{2}$

$$
C_{2}=-C_{1} \mu_{0}(t, \tau)+\frac{\partial \mu_{0}(t, \tau)}{\partial \tau}
$$

the coefficients of which are determine by background mortality $\mu_{0}(t, \tau)$ dependent on the state of environment, and by its changes in age.

The increment $\mu(t, \tau)$ are calculated from the data in Table 1 in the finite-difference form: $\partial \mu(t, \tau) \approx \Delta \mu\left(t, \tau_{j}\right)=\mu\left(t, \tau_{j+1}\right)-\mu\left(t, \tau_{j}\right), \Delta \tau_{j}=\tau_{j+1}-\tau_{j}$, where $j$ is the age group number. To estimate the connections of the variables used the correlation and regression statistical analysis. The correlation and regression coefficients largely have significance at the level $p>0.9$.

The coefficients $C_{1}, C_{2}$ of the regression equation $\frac{\Delta \mu(t, \tau)}{\Delta \tau}=C_{1} \mu(t, \tau)+C_{2}$ were estimated for population groups in the age interval 12-74 years for each year for the entire 20-year period of observations. The ageing rate of population $C_{1}=\alpha(t)$ for that time altered drastically (Fig. 2 ), with a tendency toward a decrease. Its largest values were observed until 1991 (the start of the reforms), and then till 2001 inclusive (the second stage of the reforms); there was a period of relative stabilization, and in 2002 onward there was taking place a dramatic decrease of the coefficient followed by its gradual increase.

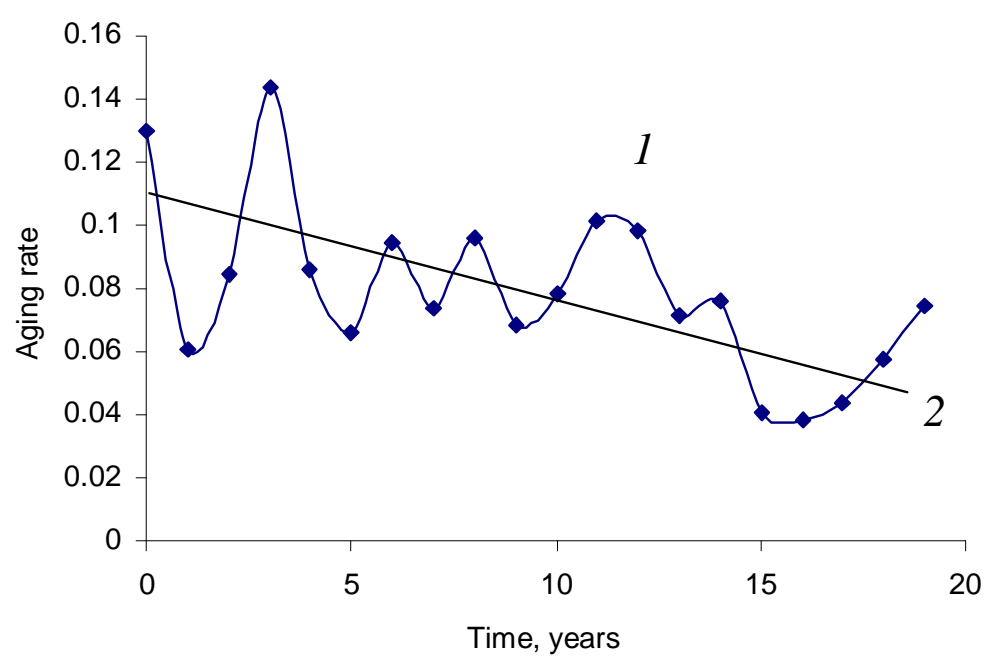

Figure 2: Time variation of the population ageing rate of a population (1) and the tendency (2) toward its decrease $\left(C_{1}=-0.0026 t+0.104, r=-0.58\right)$, time $t=0$ corresponds to 1986)

The linear dependence (3.16) of the coefficients $C_{1}$ and $C_{2}$ has the form: 


$$
C_{2}=-696.1 C_{1}+71.99, \mu_{0}(t, \tau)=696.1, \frac{\partial \mu_{0}(t, \tau)}{\partial \tau}=71.99, r^{2}=-0.90
$$

(on the interval 1986-1992);

$$
C_{2}=-1104.8 C_{1}+88.55, \mu_{0}(t, \tau)=1104.8, \frac{\partial \mu_{0}(t, \tau)}{\partial \tau}=88.55, r^{2}=-0.81
$$

(on the interval 1991-2006). The difference of the values of background mortality $\mu_{0}(t, \tau)$ prior to and after the start of the reforms is about 400 persons per year per 100000 males toward an increase. An increase of the background value of $\mu_{0}(t, \tau)$, reflecting the state of environment, according to (3.13), entails an increase of the mortality coefficient; therefore, the increase of the value of $\mu_{0}(t, \tau)$ indicates a worsening of the environmental conditions. The curve of variation of $\mu_{0}(t, \tau)$, obtained by the method of rolling regression for $C_{1}$ and $C_{2}$ for 5-year intervals, shows a tendency for change of the background mortality level (Fig. 3). This value on some time intervals is relatively stable and changes abruptly from the level of 800 to 1400 persons from the start of the reforms. During 1996-1998 there occurred a decrease in background mortality indices; however, after the 1998 financial crisis the curve $\mu_{0}(t, \tau)$ was consistently going up.

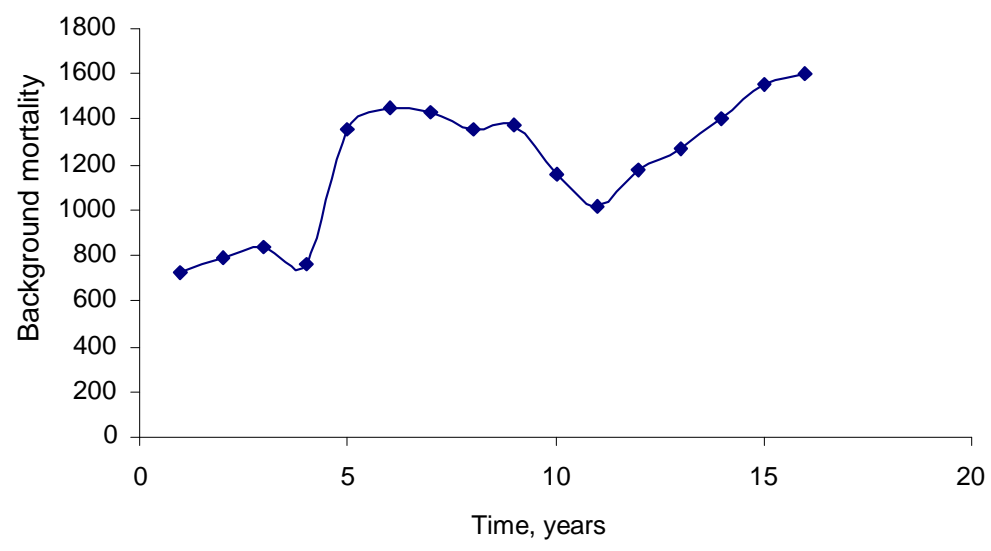

Figure 3: Time variation in background mortality (pers./year per 100000 pers.)

A comparison of the coefficients for all the 5-year intervals revealed an empirical dependence, the equation of directrix of development of the situation:

$$
\frac{\partial \mu_{0}(t, \tau)}{\partial \tau}=-k_{1} \mu_{0}(t, \tau)+k_{0}, k_{1}=-0.0513, k_{0}=35.53, r^{2}=0.88
$$

This connection is significant ( $p>0.99$ ), which confirms the validity of the expression (3.16) at constant values of $C_{2}=k_{0}, C_{1}=-k_{1}=-(c+k)$. The manifestation of the effect (3.17), i.e. an autonomous change of the state of environment (like an explosion), is explained by the fact that the fluctuation of the mortality index $\mu(t, \tau)$ in (3.16) is caused by the influence of the environment which is estimated in an integral fashion, according to (3.17), by the value of $k_{0}=$ 
$\frac{\partial \mu_{0}(t, \tau)}{\partial \tau}+(c+k) \mu_{0}(t, \tau)$. Upon substituting this expression into (3.14), we obtain an equivalent expression $k_{0}=\frac{\partial \mu(t, \tau)}{\partial \tau}+(c+k) \mu(t, \tau)$, whence

$$
\mu(t, \tau)=C_{0} \exp [-(c+k) \tau]+\frac{k_{0}}{c+k}, C_{0}-\text { const } .
$$

In this variant of the model for $\mu(t, \tau)$ there is no explicit characteristic of the environment $\mu_{0}(t, \tau)$, and the values of the coefficients $D_{1}=k_{0} /(c+k)$ and $D_{2}=C_{0}$ vary depending on the quantities forming part of it. At a constant value of $c+k$, from (3.16) for different times $t_{1}$ and $t_{2}$ we have a linear connection $\mu\left(t_{1}, \tau\right)$ and $\mu\left(t_{2}, \tau\right)$ :

$$
\mu\left(t_{2}, \tau\right)=D_{12} \mu\left(t_{1}, \tau\right)+E_{12}, D_{12}=D_{2}\left(t_{1}\right) / D_{2}\left(t_{2}\right), E_{12}=-D_{12} D_{1}\left(t_{2}\right)+D_{1}\left(t_{1}\right),
$$

where $D_{12}$ and $E_{12}$ are the transfer and correction coefficients, respectively.

Comparison of the initial data (see Table1) on the mortality intensity from the contiguous columns in the age interval 10-74 years confirms the validity of the expression (3.19). The values of the transfer coefficient $D_{12}$ vary only slightly around the value approximately equal to unity. This indicates a similarity of the values of $D_{2}$, i.e. the time variability of the form of the age dependence $\mu(t, \tau)$ does exist, even if not significant, and is determined by the variation of $c$ and $k$.

Such an analysis was also made for the temporal variability of the mortality intensity $\mu(t, \tau)$ for different ages $\tau$. The study provided similar results, yet with a lower significance $(p>0.8)$ which make it possible to identify background mortality $\mu_{0}(t, \tau)$ as an exponentially increasing function of age, with its subsequent decrease after 60 years (Fig. 4).

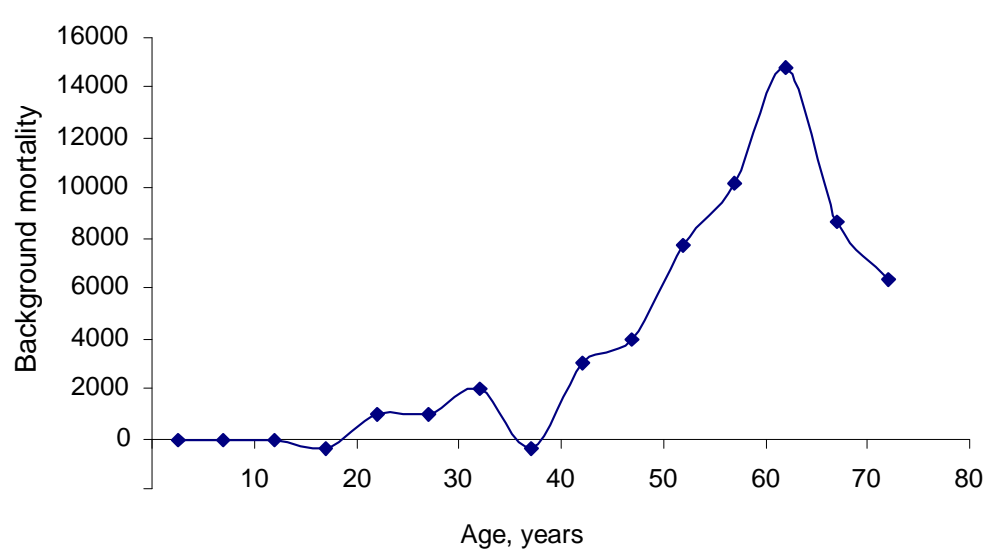

Figure 4: Age-specific variation in background mortality $\mu_{0}(t, \tau)$ (pers./year per 100000 persons).

Calculations of the regression connection of the time variation of $\mu(t, \tau)$ for adjacent age groups in the age interval 10-74 years also show the presence of a linear connection with the transfer coefficient approaching unity (1.01), on the average. A significant $(p>0.95)$ positive time dependence for age groups is observed in the able-bodied age (20-64 years); in the other groups, no tendency is pronounced, or it is negative (Fig. 5). 


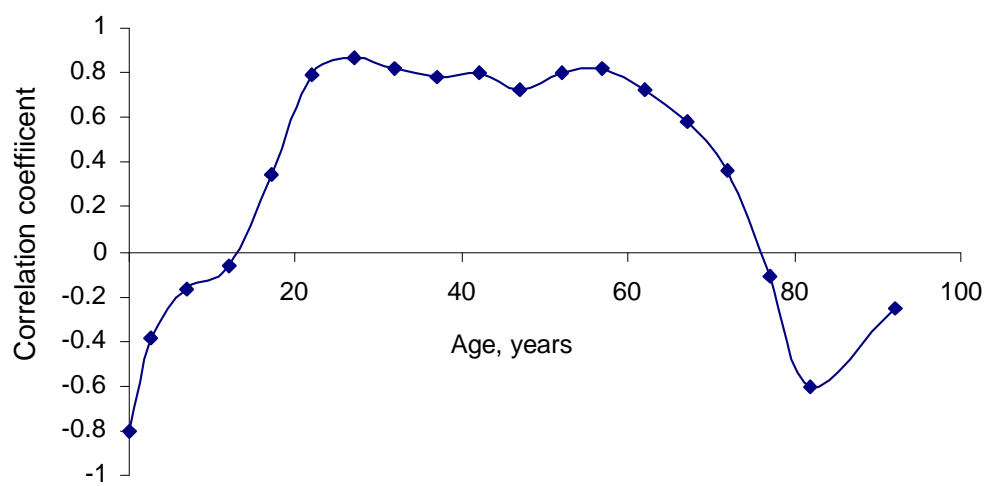

Figure 5: Time variation of the correlation coefficient of a linear dependence of the mortality rate $\mu(t, \tau)$ for age groups.

\section{Conclusion}

Polysystem investigations furnish a means of assessing from different aspects the accumulated information in order to gain insights into the hidden regularities and to explain the reasons behind the changes in substantive parameters of the models. In the study of the demographic situation, such a parameter is, among other parameters, background mortality whose value depends on a complex of factors of the state of environment. It is added to the age component of mortality and shows, in the case under consideration, a tendency toward an increase and toward a growth both with age, which is understandable from the biological standpoint, and with time. This is characteristic for the situation in the city of Angarsk associated with the dependence of the mortality indices on the state of habitat environment. Such a tendency is allowed by the solutions of differential equations taking background phenomena into account.

Through the use of different model interpretations of observed phenomena, it is possible to add to a standard dynamic model new qualities of behavior which are useful for explaining complex tendencies. In particular, the Gompert - Makeham equation arises as a consequence of existence of the homeostatic regulation process of the population size. The target state of a population depends on the multitude of influencing factors and thresholds fo their impact.

Medico-demographic monitoring data were used to verify the validity of the hypotheses of supermortality in Russia by using a model interpretation of the data. The hypotheses explain quite well the phenomena observed, which lends support to the usefulness of the hypotheses, models and data used. Two levels of influence are instructively traceable: 1) a short-lasting, factor level causing a stress response, and 2) a long-lasting level of environmental conditions which alters the dynamic stereotype.

The suggested models reflect the main aspects of the adjustment response of an urban population in answer to the disturbances of the factors and conditions of life activity, the threshold parameters of the state of the population. Stress and change in traditional conditions of existence do not mobilize any adapting processes in a population; on the contrary, they decrease its resistivity to external impacts, causing the development of varied pathological changes, accelerate ageing, 
and increase mortality. As a result, the medico-demographic status turns out to be extremely unstable, with tendencies toward an increase in mortality not only with age but also with time. This is quite well manifested in a constancy of the coefficient $k_{0}$, relating the change in characteristics of a population and its environment. As a result, change in mortality repeats the main tendencies for change of the environment which are realized in an autonomous self-developing regime of a sort.

These peculiarities in the model are explained by the individual values of the coefficients which arise in interpreting the differential equations and, judging from the data and results of analysis, are correlated with each other. They are dependent on the initial and boundary conditions that emerged at the early stage of the process, and hence are potentially amenable to regulation and management.

\section{References}

[1] S. Preston, P. Heuveline, M. Guillot. Demography: Measuring and Modeling Population Processes, Wiley-Blackwell, 2000.

[2] A. I.Yashin, I.A. Yachin, A.S.Begun. Mortality modeling: A review, Mathematical Population Studies, 4 (2000), 305 -332.

[3] S.D. Tuljapurkar. Population Dynamics in Variable Environments. Lecture Notes in Biomathematics, N 85, New York, Springer, 1990.

[4] J.L. Bobadilla, C.A. Costello, F. Mitchell (Ed.) Premature Death in the New Independent States, Washington, National Academy Press, 1997.

[5] B.T. Velichkovsky. Viability of the Nation. Role of Social Stress and Genetic Processes in a Population in the Development of a Demographic Crisis and Change of Russia's Population Health Status, Moscow, RAMN, 2008.

[6] I.A. Gundarov. Demographic Catastrophe in Russia, Moscow, URSS, 2001.

[7] Ya.A. Leshchenko. Crisis in Public Health and Socio-Demographic Development, Causes, Conditions of Overcoming, Irkutsk, East-Siberian Scientific Center of the Siberian Branch RAMS, 2006.

[8] Ya.A. Leshchenko (Ed.). Public Health as a Crucial Component of Human Capital, Irkutsk, Reproduction Center, 2005.

[9] L.A. Gavrilov, N.S. Gavrilova. The Biology of Lifetime, Moscow, Nauka, 1991.

[10] V.N. Yarygin. Health as a Biological Category: Basic mechanisms and Evolutionary Strategy, Selected Lectures for Practitioners, Moscow, 2002, 322-310. 
[11] A.K. Cherkashin. Polysystem Analysis and Synthesis. Application in Geography, Novosibirsk, Nauka, 1997.

[12] A.K. Cherkashin. Polysystem Modelling, Novosibirsk, Nauka, 2005.

[13] A.K. Cherkashin. Polysystem Modelling of Geographical Processes and Phenomena in Nature and Society, Mathematical Modelling of Natural Phenomena, 5 (2009), 4-20.

[14] D.R. Anderson. Model based Inference in the life sciences, New York-Berlin-Heidelberg, Springer Verlag, 2008.

[15] K.P. Burnham, D.R. Anderson. Model Selection and Multimodel Inference: A Practical Information Theoretic Approach, New York-Berlin-Heidelberg, 2nd ed., Springer Verlag, 2002.

[16] L. See, S. Openshaw. A hybrid multi-model approach to river level forecasting. Hydrological Sciences-Journal-des Sciences Hydrologiques, 45, 4 (2000), 523-536.

[17] T.N. Palmer, A. Alessandri, U. Andersen, P. Cantelaube, M. Davey, P.Delecluse, M. Deque, E. Diez, F.J. Doblas-Reyes, H. Feddersen, R.Graham, S. Gualdi, J.-F. Gueremy, R. Hagedorn, M. Hoshen, N. Keenlyside, M. Latif, A. Lazar, E. Maisonnave, V. Marletto, A.P. Morse, B.Orfila, P.Rogel, J.-M. Terres, M.C. Thomson. Development of a european multimodel ensemble system for seasonal-to-interannual prediction (DEMETER), Bull. Amer. Meteor. Soc., 85 (2004), 853-872.

[18] A.G. Vishnevsky (Ed.). The Population of Russia 1997. The Fifth Annual Demographic Report, Moscow, INP RAN, Center for Demography and Human Ecology, Moscow, 1998.

[19] B.T. Velichkovsky. Social Stress, Labor Motivation and Health, Moscow, Zashchita, 2005.

[20] V. Shkolnikov, E. Andreyev, T. Moleva (Eds). Inequality and Mortality in Russia, Moscow, Carnegie Moscow Center, 2000.

[21] L.L. Rybakovsky. Applied Demography, Moscow, ISPI RAN, 2003.

[22] V.N. Rudenko. The demographic crisis in Russia: causes and consequences, Vestnik Uralskogo otdeleniya RAN, 1 (2007), 103-118.

[23] A.V. Shafirkin. Influence of chronic psychoemotional stress on population health, Avikosmicheskaya i ekologicheskaya meditsina, 3 (2003), 31-38.

[24] E.A. Tishuk. Some questions concerning health status of the population of the Russian Federation, Problemy sotsialnoi gigieny, zdravookhraneniya i istorii medistiny, 6 (2001), 3-8.

[25] W.C. Cockerham. Health and social change in Russia and eastern Europe, New York, Routledge, 1999.

[26] V. Chkolnikov, M. McKee, D.A. Leon. Changes in life expectancy in Russia in the mid-1990s, Lancet, 357 (2001), 917-921. 
[27] P.V. Simonov. The Emotional Brain, Moscow, Nauka, 1981.

[28] P.V. Simonov. The necessities-and-information theory of emotions, Voprosy psikhologii, 6 (1982), 44-56.

[29] J. Shen. On the foundations of vision modeling : I. Weber's law and Weberized TV restoration, Physica D: Nonlinear Phenomena, 3-4 ( 2003), 241-251. 